\title{
Hepatectomia videolaparoscópica com dispositivo de radiofrequência
}

\section{Laparoscopic hepatectomy with radiofrequency device}

\author{
Vivian Resende, TCBC-MG ${ }^{1}$; Cristiano Xavier Lima²; Mário Marcos lusckal³; Milton Carlos Aguiar Junior ${ }^{4}$; Emanuele Gammeri \\ Nagy Adley Habib ${ }^{6}$; José Renan da Cunha-Melo ${ }^{7}$
}

\section{RE S U M O}

\begin{abstract}
As ressecções hepáticas laparoscópicas vêm ganhando adeptos entre cirurgiões pois evidenciam recuperação rápida, menor permanência hospitalar e melhor resultado estético. A utilização do dispositivo laparoscópico de radiofreqüência foi pela primeira vez utilizada, com sucesso, no Brasil, para a ressecção de carcinoma hepatocelular no segmento VI, em dois pacientes cirróticos. Apesar do sangramento intraoperatório continuar sendo um grande desafio para o cirurgião durante ressecções hepáticas laparoscópicas, em ambos os casos, a exclusão vascular hepática foi dispensável e não houve necessidade de hemotransfusão. Os pacientes receberam alta hospitalar no quarto dia pós-operatório.
\end{abstract}

Descritores: Cirrose hepática. Carcinoma hepatocelular. Hepatectomia. Cirurgia videoassistida. Dispositivo de identificação por radiofrequência.

INTRODUÇÃO

sangramento intraoperatório durante ressecções hepáticas continua a ser grande desafio para o cirurgião. Aparelhos, como dissector ultrassônico (CUSA), bisturi harmônico, pinça bipolar e radiofreqüência, são atualmente disponíveis para ajudar na secção do parênquima hepático. A manobra de Pringle é algumas vezes utilizada como medida para diminuir o sangramento.

As ressecções hepáticas laparoscópicas vem ganhando adeptos entre cirurgiões pois evidenciam uma rápida recuperação pós operatória, menor permanência hospitalar, além de melhor resultado estético ${ }^{1,2}$. O controle intraoperatório do sangramento durante procedimento laparoscópico pode ser muito difícil e algumas vezes resulta em conversão para cirurgia aberta. Desde a descrição inicial de ressecção hepática laparoscópica utilizando radiofreqüência um novo dispositivo para uso laparoscópico tem sido desenvolvido e utilizado 1,3,4.

O objetivo da presente nota é descrever a técnica para ressecção hepática laparoscópica utilizando-se, pela primeira vez no Brasil, o dispositivo de radiofreqüência, em pacientes cirróticos portadores de carcinoma hepatocelular.

\section{DESCRIÇÃO TÉCNICA}

Foram dois pacientes admitidos no Grupo de Fígado do Instituto Alfa de Gastroenterologia da Universidade Federal de Minas Gerais. O Primeiro deles do sexo feminino, 69 anos, portadora de cirrose pós viral $\mathrm{C}$ e colecistolitíase. O segundo, masculino, 63 anos, com cirrose pós-viral B. Ambos eram Child Pugh A, sem sinais de hipertensão porta significativos e sem critérios para o transplante hepático. Apresentavam lesão no segmento VI do fígado que media $7 \mathrm{~cm} \times 5 \mathrm{~cm}$ e $5 \mathrm{~cm} \times 4 \mathrm{~cm}$, respectivamente. Em ambos, a tomografia de abdome com contraste endovenoso e a ressonância nuclear magnética foram compatíveis com carcinoma hepatocelular (Figuras 1a e 1b). A dosagem sérica de alfafetoproteina estava normal. Um deles tinha comorbidade (bócio mergulhante que atingia a bifurcação da traquéia).

Realizou-se ressecção laparoscópica utilizandose o dispositivo laparoscópico, que é formado por um arranjo de quatro agulhas dispostas de forma retangular e que utilizam a energia de radiofreqüência ${ }^{1,3}$. Após anestesia geral o paciente foi colocado em decúbito lateral esquerdo. Realizou-se punção e pneumoperitônio que foi manti-

\footnotetext{
Trabalho realizado no Instituto Alfa de Gastroenterologia do Hospital das Clínicas da Universidade Federal de Minas Gerais-MG-BR.

1. Professora Adjunta do Departamento de Cirurgia da Universidade Federal de Minas Gerais (UFMG); 2. Professor Adjunto do Departamento de Cirurgia da Universidade Federal de Minas Gerais (UFMG); 3.: Médico Residente (R4) em Cirurgia do Aparelho Digestivo do Instituto Alfa de Gastroenterologia (IAG) do Hospital das Clínicas da UFMG; 4. Médico Residente (R4) em Cirurgia do Aparelho Digestivo do Instituto Alfa de Gastroenterologia (IAG) do Hospital das Clínicas da UFMG; 5. Médico Residente da Universidade de Messina (Sicília); 6. Chefe do Serviço de Cirurgia Hepatobiliopancreática do Hospital Hammersmith, Imperial College of London; 7. Professor. Titular do Departamento de Cirurgia da Universidade Federal de Minas Gerais (UFMG).
} 

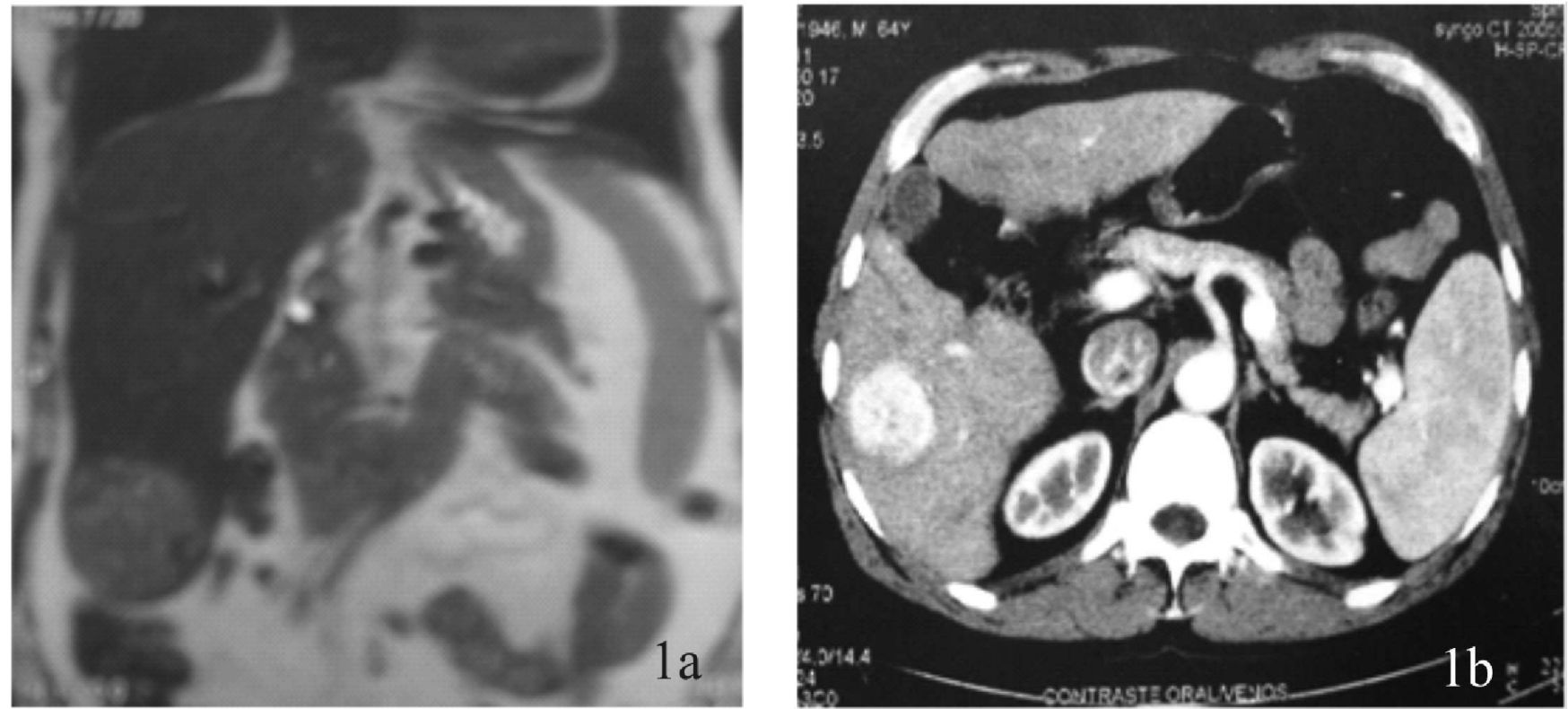

Figura 1 - Imagens de carcinoma hepatocelular no segmento VI do fígado (setas), em dois pacientes submetidos à ressecção laparoscópica. 1a: ressonância magnética do abdome (corte frontal); 1b: angiotomografia de abdome (corte transversal).

do com $\mathrm{CO}_{2}$ numa pressão de $12 \mathrm{mmHg}$. Foram colocados três portais de $10 \mathrm{~mm}$ e dois de $5 \mathrm{~mm}$.

Após secção do ligamento hepatorrenal, demarcou-se com o eletrocautério, uma linha na superfície superior e inferior do fígado delimitando-se a área do segmento VI a ser ressecada (Figura 2a). Aplicou-se o dispositivo, perpendicularmente, ao longo desta linha em ambas as superfícies marcadas (Figuras 2a e 2b). Realizou-se uma segunda linha de ablação no parênquima, correspondente ao lado da lesão, que levou à completa isquemia do segmento VI. Em seguida, o dispositivo foi, sucessivamente, aplicado paralelamente às linhas de ablação perpendicular (Figura 2c), à medida que o parênquima isquêmico foi seccionado com tesoura laparoscópica (Figura 2d). Ao término do procedimento, a área de secção do parênquima ficou completamente regular, sem extravazamento de bile ou sangue (Figura 2e). Colocou-se dreno tubular pelo orifício de um dos portais laterais (Figura 2f).

Durante todo o procedimento, não houve necessidade de exclusão vascular hepática. Colecistectomia foi realizada em um dos pacientes. A peça cirúrgica foi colocada em bolsa plástica e retirada através do portal umbilical, que teve a sua incisão ampliada.

A duração do procedimento cirúrgico foi em média 120 minutos. A perda sanguínea média foi de $50 \mathrm{ml}$ nos dois pacientes. Nenhum deles necessitou de hemotransfusão.

Ambos evoluíram sem complicações e receberam alta hospitalar no quarto dia pós-operatório. O exame anatomopatológico mostrou carcinoma hepatocelular bem diferenciado, com invasão vascular e margens cirúrgicas livres.

\section{DISCUSSÃO}

Recomenda-se uma cuidadosa seleção dos pacientes candidatos à ressecção laparoscópica do fígado. Apesar de resseções maiores já terem sido descritas por laparoscopia ${ }^{5}$, esta técnica é idealmente considerada para ressecção de tumores menores localizados perifericamente em segmentos inferiores ou laterais do fígado. Tumores grandes localizados profundamente ou próximos à bifurcação porta ou, ainda, nos segmentos posteriores não são usualmente selecionados para ressecção laparoscópica 1,2,4.

O dispositivo laparoscópico "Habib Sealer 4X" ( LHS, Angiodynamics, Queensbury, NY), mede $45 \mathrm{~cm}$ de comprimento com uma parte isolada que permite a aplicação de radiofreqüência numa extensão de $5 \mathrm{~cm}$. Um botão de controle no cabo permite ao cirurgião escolher a profundidade da ablação. O aparelho pode ser introduzido através de portal de $10 \mathrm{~mm}^{1,3}$.

Algumas vantagens da ressecção laparoscópica incluem menor dor pós-operatória, mobilização precoce e menor permanência hospitalar ${ }^{1-5}$. No presente relato os dois pacientes receberam alta hospitalar no quarto dia pósoperatório.

Recomenda-se a realização de ultrassom laparoscópico intra-operatório para a demarcação da margem cirúrgica ${ }^{1,4}$, porém, este aparelho ainda é indisponível em nossa Instituição. Entretanto, a identificação macroscópica do tumor na superfície do segmento VI permitiu a delimitação da linha de secção com segurança. O exame anatomopatológico da peça cirúrgica confirmou a inexistência de neoplasia no tecido hepático adjacente à linha de coagulação (margem cirúrgica de $2 \mathrm{~cm}$ ). Uma van- 

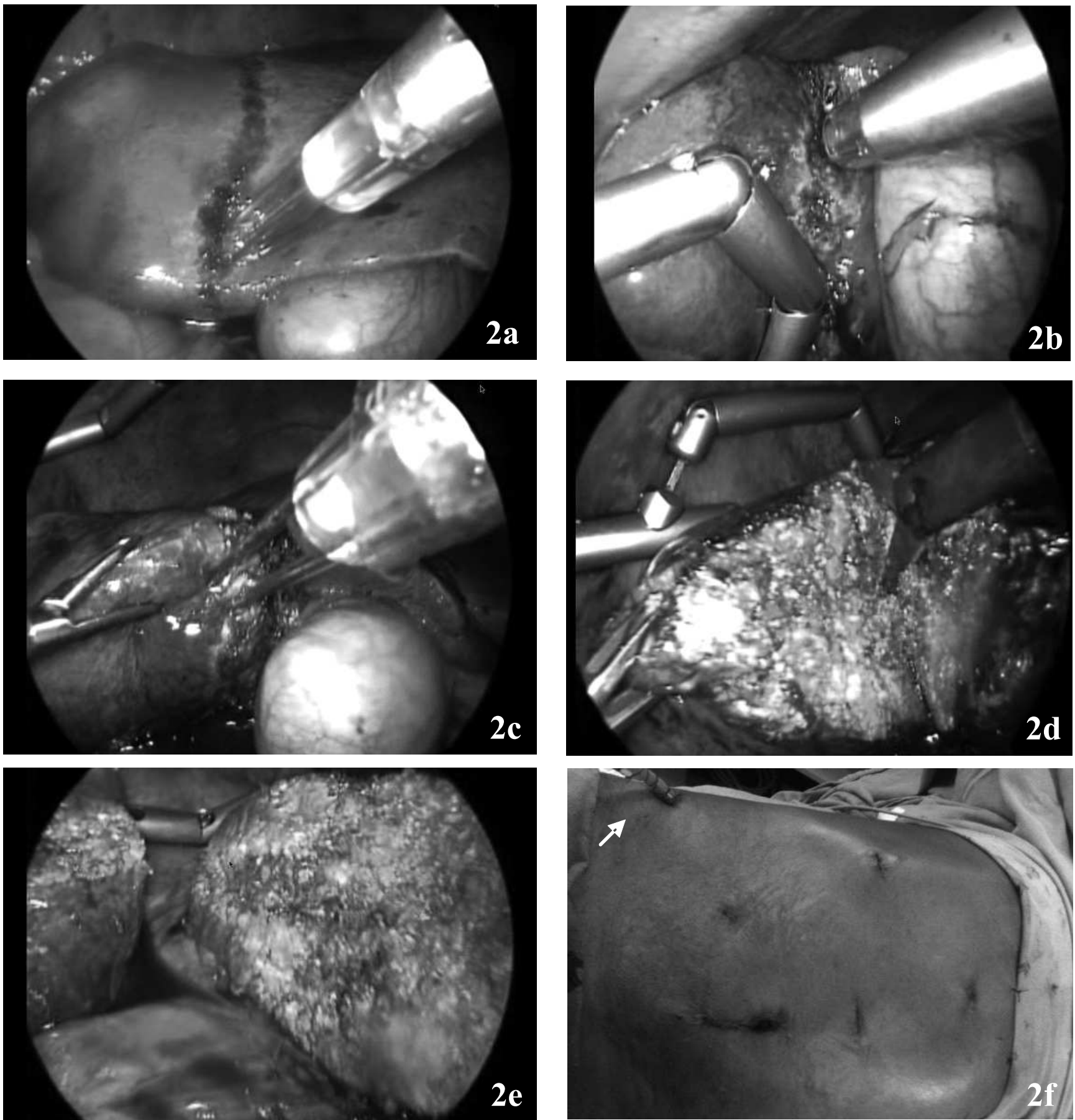

Figura 2 - Imagens de hepatectomia laparoscópica do segmento VI para tratamento de carcinoma hepatocelular. 2a: demarcação da linha de ressecção e aplicação perpendicular do dispositivo (primeira linha de ablação); $2 b$ : segunda linha de ablação vista pela face visceral do fígado. 2c: ablação paralela à linha de secção, observar a coloração isquêmica do segmento VI. 2 d: secção do parênquima hepático com tesoura laparoscópica; 2e: aspecto final da superfície de ressecção; $2 f$ : posição dos trocartes e dreno (seta).

tagem da ressecção hepática laparoscópica por meio de radiofreqüência é que, além da margem alcançada na peça cirúrgica, esta se estende à superfície de ressecção que sofre ablação ${ }^{1,3,4}$.

Dificuldades técnicas ou sangramento intra-operatório são motivos frequentes de conversão para cirurgia aberta ${ }^{2-4}$. Com o dispositivo laparoscópico por radiofreqüência a perda sanguínea intra-operatória foi mínima o que tornou a cirurgia facilmente exequível e rápida. O procedimento, entretanto, deve ser realizado por equipe com experiência em cirurgia hepatobiliopancreática e videolaparoscópica, para dimimuir morbidade e mortalidade ${ }^{6}$.

A seleção apropriada dos pacientes e técnica cirúrgica refinada foram primordiais para o sucesso da ressecção hepática laparoscópica, utilizando-se o dispositivo de radiofrequência. 


\section{A B S S T R A C T}

Laparoscopic liver resections are gaining adherents among surgeons, as they show rapid recovery, shorter hospital stay and better cosmetic results. The use of a laparoscopic radiofrequency device was first carried out successfully in Brazil for resection of hepatocellular carcinoma of the segment VI in two cirrhotic patients. Although intraoperative bleeding remains a major challenge for the surgeon during laparoscopic liver resections, in both cases the hepatic vascular exclusion was expendable and there was no need for blood transfusion. Patients were discharged on the fourth postoperative day.

Key words: Cirrhosis. Hepatocellular carcinoma. Hepatectomy. Video-assisted surgery. Radio frequency identification device.

\section{REFERENCIAS}

1. Jiao LR, Ayav A, Navarra G, Sommerville C, Pai M, Damrah O, et al. Laparoscopic liver resection assisted by the laparoscopic Habib sealer. Surgery. 2008;144(5):770-4.

2. Bryant R, Laurent A, Tayar C, Cherqui D. Laparoscopic liver resection-understanding its role in current practice: the Henri Mondor Hospital experience. Ann Surg. 2009;250(1):103-11.

3. Pai M, Navarra G Ayav A, Sommerville C, Khorsandi SK, Damrah O, et al. Laparoscopic Habib 4X: a bipolar radiofrequency device for bloodless laparoscopic liver resection. HPB. 2008;10(4):261-4.

4. Bachellier P, Ayav A, Pai M, Weber JC, Rosso E, Jaeck D, et al Laparascopic liver resection assisted with radiofrequency. Am J Surg. 2007;193(4):427-30.

5. Machado MAC, Makdissi FF, Surjan RCT, Oliveira AC, Pilla VF, Teixeira AR. Trisegmentectomia hepática direita por videolaparoscopia. Rev Col Bras Cir. 2008;35(5):338-41.

6. Resende V, Rezende-Neto JB, Fernandes JS, Cunha-Melo JR. Avaliação da morbidade e da mortalidade após ressecções hepáticas. Rev Col Bras Cir. 2011;38(5):323-6.
Recebido em 15/03/2012

Aceito para publicação em 10/04/2012

Conflito de interesse: nenhum

Fonte de financiamento: nenhuma.

\section{Como citar este artigo:}

Resende V, Lima CX, Lusckal MM, Aguiar Júnior MC, Gammeri E, Habib NA, Cunha-Melo JR. Hepatectomia videolaparoscópica com dispositivo de radiofrequência. Rev Col Bras Cir. [periódico na Internet] 2013;40(2). Disponível em URL: http://www.scielo.br/rcbc

\section{Endereço para correspondência:}

Vivian Resende

E-mail: vivianresen@gmail.com 